\title{
DIMENSION LOWERING MAPPINGS OF CONVEX SETS
}

\section{R. H. SORGENFREY}

It is a well known result of Borsuk [2] that any mapping ${ }^{2}$ of the $n$-sphere $S_{n}$ into euclidean $n$-space carries some two antipodal points of $S_{n}$ into the same point; that is, the inverse of some point has the same diameter as $S_{n}$. It is the principal purpose of this paper to obtain results concerning the diameter of connected subsets of such inverse sets when convex sets are mapped into spaces of lower dimension. The first part of the paper concerns itself with convex sets of dimension greater than two; in the second part somewhat sharper results are obtained for mappings of plane convex sets.

1. There will first be obtained the following rather trivial extension of Borsuk's theorem to convex sets.

TheOREM 1. If $K$ is a compact convex set of dimension $n$, and $f$ maps $K$ into euclidean space $E$ of dimension $n-1$, then the inverse of some point of $E$ has diameter at least $w$, the width ${ }^{3}$ of $K$.

Proof. Since for each $\epsilon>0, K$ contains a convex set of width $w-\epsilon$ with the property that each supporting hyperplane intersects its boundary in a single point, it may be assumed for purposes of this proof that $K$ itself has this property. For each unit vector $\boldsymbol{u}$, let $g(\boldsymbol{u})$ be the point of contact of the oriented supporting hyperplane normal to $\boldsymbol{u}$. Then $f(g(u))$ is a mapping of the $n-1$ sphere into $E$, and by Borsuk's theorem there is a $u_{0}$ such that $f\left(g\left(u_{0}\right)\right)=f\left(g\left(-u_{0}\right)\right)=y_{0} \in E$. Hence $g\left(u_{0}\right), g\left(-u_{0}\right) \in f^{-1}\left(y_{0}\right)$, and since the distance from $g\left(u_{0}\right)$ to $g\left(-u_{0}\right)$ is at least $w$, the proof is complete.

TheOREM 2. If $K$ is a compact convex set of dimension $n(n>3)$, and $f$ maps $K$ into a set $F$ of dimension $m \leqq(n-2) / 2$, then the inverse of some point of $F$ has a connected subset of diameter at least w, the width of $K$.

Proof. Let $f=h g$ be the monotone-light factorization of $f[4, \mathrm{p}$. 141]; that is, $g(K)=D$ is monotone, $h(D)=F$ is light, and $f(x)$ $=h(g(x))$ for all $x \in K$. Since for each $z \in F, h^{-1}(z)$ is closed and totally

Presented to the Society, May 3,1952, under the title Mappings of plane convex sets; received by the editors June 18, 1953.

${ }_{1}$ Numbers in brackets refer to the bibliography at the end of the paper.

2 Throughout this paper the term mapping will mean continuous transformation.

${ }^{3} \mathrm{By}$ the width of a convex set is meant the least distance between parallel supporting planes. 
disconnected, and therefore zero-dimensional, it follows [3, p. 91] that $D$ has dimension $m$. Consequently $[3$, p. 56] $D$ can be embedded in euclidean space of dimension $2 m+1 \leqq n-1$. Hence, by Theorem 1 , there is a $y_{0} \in D$ such that diam $g^{-1}\left(y_{0}\right) \geqq w$. But $g^{-1}\left(y_{0}\right)$ is connected and is a subset of $f^{-1}\left(h\left(y_{0}\right)\right)$ and the theorem follows.

REMARK. If $n=3$ and $m=1$, Theorem 2 remains true; in this case $D$ is a dendrite (see proof of Lemma 2) and is therefore imbeddable in the euclidean plane. Otherwise the question for $n>m>(n-2) / 2$ remains undecided, except for the case $n=2$, which is treated in the next section.

2. Lemma 1. If $C$ is a circular disc of diameter $d$, and $f$ maps $C$ into $a$ one-dimensional set $F$, then the inverse of some point of $F$ contains a connected set of diameter at least $d 3^{1 / 2} / 2$.

PRoOF. Let $f=h g$ be the monotone-light factorization of $f: g(C)$ $=D$ is monotone, $h(D)=F$ is light. Just as in the proof of Theorem 2, it follows that $D$ is one-dimensional. Also, since it is the monotone continuous image of a locally connected, unicoherent continuum, $D$ has these properties [4, p. 33 and p. 138]. Since it is a one-dimensional, unicoherent locally connected continuum, $D$ is a dendrite (=acyclic curve). Let $p, q$, and $r$ be points equally spaced on the boundary $B$ of $C$; dist $(p, q)=\operatorname{dist}(q, r)=\operatorname{dist}(r, p)=d 3^{1 / 2} / 2$. If $g(p)=g(q)$, then $g^{-1}(g(p))$ has diameter at least $d 3^{1 / 2} / 2$. If $g(p) \neq g(q)$, then there is a unique $\operatorname{arc} A$ in $D$ joining these two points. Suppose that for each $y \in A$, diam $g^{-1}(y)<d 3^{1 / 2} / 2$. Since any set which intersects all three of the closed $120^{\circ}$ arcs $p q, q r$, and $r p$ has diameter at least $d 3^{1 / 2} / 2$, it follows that for each $y \in A, g^{-1}(y)$ intersects at most two of these. But since each $y \in A, y \neq g(p), y \neq g(q)$, separates $g(p)$ from $g(q)$ in $D$, it follows that $g^{-1}(y)$ separates $p$ and $q$ in $C$; hence for each $y \in A$, $g^{-1}(y)$ intersects $p q$ and just one of $q r$ and $r p$. Denote by $P$ the set of all $y \in A$ such that $g^{-1}(y)$ intersects $r p$ and by $Q$ the set of all $y \in A$ such that $g^{-1}(y)$ intersects $q r$. It is easily seen that $P$ and $Q$ are nonvoid, disjoint, and closed, and that they therefore form a separation of $A=P \cup Q$. This contradiction establishes the fact that for some $y_{0} \in D, g^{-1}(y)$, which is a connected set, has diameter at least $d 3^{1 / 2} / 2$. It follows that $f^{-1}\left(h\left(y_{0}\right)\right)$ contains a connected set of diameter at least $d 3^{1 / 2} / 2$, and the lemma is established.

TheOREM 3. If $K$ is a compact plane convex set of width w, andf maps $K$ into a one-dimensional set $F$, then the inverse of some point of $F$ contains a connected set of diameter at least $w 3^{1 / 2} / 3$.

Proof. The set $K$ contains a circular disc $C$ of diameter $2 w / 3[1$, p. 53]. By Lemma 1, the inverse of some point under the partial 
mapping $f \mid C$ contains a connected set of diameter at least $(2 w / 3) 3^{1 / 2} / 2=w 3^{1 / 2} / 3$, and the theorem is proven.

Theorem 4 . If $K$ is a compact, centrally symmetric, plane convex set of width w, and $f$ maps $K$ into a one-dimensional set $F$, then the inverse of some point of $F$ contains a connected set of diameter at least w3/2/2.

PROof. Since a centrally symmetric convex set of width $w$ contains a circle of diameter $w$, the theorem is an immediate consequence of Lemma 1.

That the results established in Theorems 3 and 4 are the best possible is shown by the following examples.

EXAmple 1. Let $K$ be an equilateral triangle ${ }^{4}$ of side 2. Let its vertices be $p_{1}, p_{2}$, and $p_{3}$ and let $q_{i}$ be the mid-point of the side opposite $p_{i}$. Denote by $G_{1}$ the collection consisting of $\left\{p_{1}\right\}$ and the intervals, other than $q_{2} q_{3}$, which are intersections of lines parallel to $q_{2} q_{3}$ with the triangle $p_{1} q_{2} q_{3}$. Define the collections $G_{2}$ and $G_{3}$ similarly. Then the collection $G$ consisting of the triangle $q_{1} q_{2} q_{3}$ and the elements of $G_{1}, G_{2}$, and $G_{3}$ is upper semi-continuous and fills up $K$. Consequently there is a mapping $f$ carrying $K$ into a triod $T$ such that the elements of $G$ are the inverses of points of $T$. It is easily verified that the width $w$ of $K$ is $3^{1 / 2}$ and that the largest diameter $d$ of the inverse of a point of $T$ is 1 . Hence $d=w 3^{1 / 2} / 3$, which shows that the result obtained in Theorem 3 cannot be improved.

Example 2. In Example 1 let $K^{\prime}$ be the circular disc inscribed in $K$, and let $f^{\prime}=f \mid K^{\prime}$. The width $w^{\prime}$ of $K^{\prime}$ is $2 / 3^{1 / 2}$ and the largest diameter $d^{\prime}$ of the inverse of a point under $f^{\prime}$ is, as before, 1. Then $d^{\prime}=w^{\prime} 3^{1 / 2} / 2$, which shows that the result obtained in Theorem 4 for centrally symmetric sets is the best possible.

Remark. The conclusions of Theorems 3 and 4 cannot be improved even if $f$ is real-valued since there is a simple mapping of the triod of the above examples into the unit interval which sends the junction point into 0 and the end points into 1 .

\section{BIBLIOGRAPHY} 1934.

1. T. Bonnesen and W. Fenchel, Theorie der Konvexen Körper, Berlin, Springer,

2. K. Borsuk, Drei Sätze über die n-dimensionale euklidische Sphäre, Fund. Math. vol. 20 (1933) pp. 177-190. 1948.

3. W. Hurewicz and H. Wallman, Dimension theory, Princeton University Press,

4. G. T. Whyburn, Analytic topology, Amer. Math. Soc. Colloquium Publications, vol. 28, 1942.

University of California, Los ANgeles

${ }^{4}$ In these examples, by triangle is meant triangular lamina. 